\title{
Melanotan gegen die blasse Winterhaut?
}

\section{Melanotan Against Fair Winter Skin?}

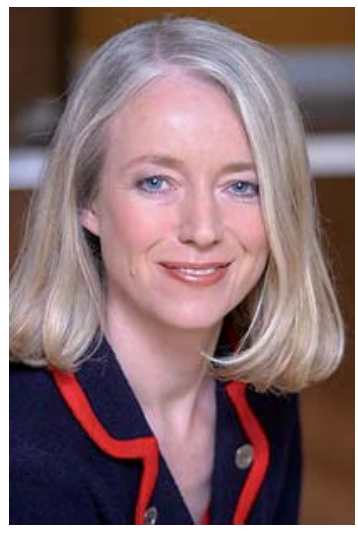

Prof. Dr. Christiane Bayerl
Bibliografie

Dol http://dx.doi.org/ 10.1055/s-0030-1256274

Akt Dermatol 2011; 37; 63

(c) Georg Thieme Verlag KG .

Stuttgart · New York

ISSN 0340-2541

Korrespondenzadresse

Prof. Dr. med. Christiane Bayerl

Klinik für Dermatologie

und Allergologie

HSK, Wilhelm-Fresenius-Klinik

Städtisches Klinikum Wiesbaden

Aukammallee 39

65191 Wiesbaden

christiane.bayer!@

hsk-wiesbaden.de
Der Winter ist vorbei und die blasse Winterhaut kommt ans Tageslicht und muss nicht mehr mit dicken Pullovern, Strümpfen und langen Unterhosen gewärmt werden. Wem fehlt da nicht ein diskreter Braunton? Dann würde man doch viel gesünder und vitaler aussehen! Menschen, die dieser Meinung sehr intensiv anhängen, werden in der flapsigen Formulierung als „Bronzoholics“ oder „Tanorektiker“ bezeichnet. Damit ist gemeint, dass das Sonnenbad einen Genuss darstellt, der bereits zur Abhängigkeit geführt hat. Typischerweise wird die Sonne dann wie eine Droge genossen. Vernünftige Gegenargumente wie die Hauttumorentstehung und die Hautalterung sind den „Abhängigen“ zwar bekannt, führen aber nicht zu einer Änderung des Sonnenverhaltens.

Und dann kam der Ausweg, Melanotan, die „BarbieDroge“ oder auch „Ken-Droge“ genannt. Die Substanz fördert als Strukturverwandte des Melanozyten stimulierenden Hormons ( $\alpha$-MSH) den Gehalt an Melanin in der Haut und führt in der Tat meistens zur Bräunung. Die Substanz wurde in den 80er-Jahren am Health Science Center der University of Arizona von Dr. N. Levine entwickelt. Sie war für die Hautkrebsprophylaxe bei hellhäutigen Menschen gedacht. Entsprechend erfolgte die weitere Forschung im UV-reichen Australien. Eine Phase-IIIStudie für die Anwendung bei polymorpher Lichtdermatose läuft.

Die Substanz kann über das Internet bezogen werden, Melanotan I und II werden angeboten. Die Substanzen sind als nasale Applikatoren, als Spray, als subkutane Spritzen, als Kapseln und als Implantat, das unter die Haut injiziert wird und dann langsam den Wirkstoff freigibt, zu erhalten. Ist dieser Ausweg des vorgeblich ungefährlichen Bräunens eine Sackgasse? Das Bundesinstitut für Arzneimittel und Medizinprodukte hat in seiner Pressemitteilung vom 28. Oktober 2010 dringend davon abgeraten, aus unbestimmbaren Internetquellen die melanotanhaltigen Produkte zu beziehen. Konkret finden sich in dieser Mitteilung Erkrankungen des Herz-Kreislaufsystems, des Verdauungstraktes und das Injektionsrisiko bei Selbstinjektion des Melano-
tan-Implantats aufgeführt. Unbehelligt davon sind die klinischen Prüfungen zum Melanotan I (Afamelanotid oder NDP- $\alpha-\mathrm{MSH}$ ), einem synthetischen Peptidhormon, bei der erythropoetischen Protoporhphyrie. Im Vergleich zum $\alpha-\mathrm{MSH}$ sind zwei Aminosäuren ausgetauscht, wodurch die Substanz wirksamer sein soll als das natürliche $\alpha-M S H$. Die US amerikanische Zulassungsbehörde FDA hat Melanotan I als „Orphan Drug“ anerkannt.

Die Problematik beim privaten Kauf des Produktes ist die mangelnde Reinheit der angebotenen Substanz. Zudem soll das Nasesnspray wenig wirksam sein. Angaben aus dem Internet zufolge kommen die Hersteller der Produkte mit oft geringer Reinheit aus den USA oder China und ein illegaler Handel entwickelte sich. Die erste FDA-Warnung an die verkaufenden Firmen auf dem Markt erfolgte bereits 2007 und erneut 2009. Bei den deutschsprachigen Blogs im Internet aus der Lifestyle-Szene, vor allem unter Sport-Studio-Besuchern, wird die Substanz gelobt, einmal aufgrund der Gewichtsreduktion und dann auch aufgrund der positiven Effekte auf die Libido über zentrale Reize im Hypothalamus. Ein Autor wertet die Substanz für sich als weniger gesundheitlich gefährdend als Viagra/ Cialis etc. In der Tat liefen vor mehr als 10 Jahren Studien mit Melanotan II, einem zyklischen Peptid aus sieben Aminosäuren, mit einer $\alpha$-MSH-ähnlichen Wirkung. Als „Nebenwirkung“ wurde neben Übelkeit, Streck- und Gähnbedürfnis in einer sehr kleinen Gruppe von 3 Probanden eine spontane Erektion bemerkt. Daraufhin beschrieb die Laienpresse die Substanz als Viagra-Ersatz. Herstellung und Vertrieb ohne Zulassung starteten damals. Analog der FDA haben auch britische und skandinavische Behörden Warnungen ausgesprochen. Einige der Melanotan-Bräunungs-Internetseiten sind aktuell nicht mehr zugängig und „under construction“. Die Hauptumschlagspunkte sind Sonnenstudios und Body-Building-Einrichtungen.

Ihre

Christiane Bayerl 